Article

\title{
Substance Use among Youth in Community and Residential Mental Health Care Facilities in Ontario, Canada
}

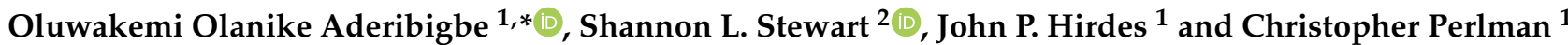 \\ 1 School of Public Health Sciences, University of Waterloo, Waterloo, ON N2L 3G1, Canada; \\ hirdes@uwaterloo.ca (J.P.H.); chris.perlman@uwaterloo.ca (C.P.) \\ 2 School and Applied Child Psychology, Faculty of Education, University of Western Ontario, \\ London, ON N6G 1G7, Canada; sstewa24@uwo.ca \\ * Correspondence: ooaderibigbe@uwaterloo.ca
}

check for updates

Citation: Aderibigbe, O.O.; Stewart, S.L.; Hirdes, J.P.; Perlman, C. Substance Use among Youth in Community and Residential Mental Health Care Facilities in Ontario, Canada. Int. J. Environ. Res. Public Health 2022, 19, 1731. https:// doi.org/10.3390/ijerph19031731

Academic Editor: Paul B.

Tchounwou

Received: 27 December 2021

Accepted: 30 January 2022

Published: 2 February 2022

Publisher's Note: MDPI stays neutral with regard to jurisdictional claims in published maps and institutional affiliations.

Copyright: (C) 2022 by the authors. Licensee MDPI, Basel, Switzerland. This article is an open access article distributed under the terms and conditions of the Creative Commons Attribution (CC BY) license (https:// creativecommons.org/licenses/by/ $4.0 /)$.

\begin{abstract}
There is a need to improve the integration of substance use and mental health care for children and youth. This study examines risk and protective factors for substance use among youth with mental health conditions who received community-based or residential care services between 2012-2020 in Ontario, Canada. In this study, a cross-sectional design was used to examine patterns and factors associated with substance use among youth (12-18 years) assessed in the community ( $\mathrm{n}=47,418)$ and residential $(\mathrm{n}=700)$ mental health care facilities in Ontario, Canada. Youth were assessed with the interRAI Child and Youth Mental Health Assessment (ChYMH). Substance use is identified by any substance use (including alcohol) 14 to 30 days prior to assessment. Logistic regression with generalized estimating equations was used to examine clinical, psychosocial, and environmental factors associated with substance use. This study shows that $22.3 \%$ of youth reported the use of substances in the community settings and $37 \%$ in residential settings. Older age group (Youth older than 16 years), being a victim of abuse, having experienced self-injurious ideation/attempt, being at risk of disrupted education, and having a parent/caregiver with addiction or substance use disorder were significantly associated with substance use. Several factors reduced the risk of substance use, including being a female, having anxiety symptoms, and having cognition problems. In conclusion, the study found that individual and parental factors increase youth's risk of substance use, highlighting the importance of a holistic approach that includes consideration of social and biological risk factors to prevention/risk reduction, risk assessment, management, and recovery.
\end{abstract}

Keywords: substance use; youth; mental health; biopsychosocial; interRAI; assessment

\section{Introduction}

Substance use disorders are one of the three most common types of mental illnesses experienced by Canadians, with young people aged 15-24 years having the highest rates of substance abuse or dependence [1]. Initiation of substance use may include experimentation or heavier and higher risk patterns of use [2-4]. Alcohol has been the most commonly used substance among youth, followed by tobacco, cannabis, and opioids $[5,6]$. School surveys in Canada have reported that up to $60 \%$ of students in grades 7-9 reported lifetime use of various substances $[7,8]$. In Ontario, Canada, $75 \%$ of youth in grade 12 reported lifetime alcohol use, $26 \%$ cannabis, and $26 \%$ nicotine [6]. Another study that examined current users of substances (use of substances in the last 30 days) shows that among youth in grades 7 to 12 in a general Canadian population sample, 27\% reported current use of alcohol, 19\% reported cannabis use, and $8 \%$ reported other drugs [9]. Substance use among youth can have a cumulative impact, contributing to costly social, physical, and mental health problems $[2-4,10,11]$. Substance use conditions are associated with problems such as poor academic performance, job instability, teen pregnancy, the transmission of sexually transmitted diseases, accidents, crimes, violence, overdoses, drug tolerance effects, 
withdrawal symptoms, and other longer-term physical health issues [11-13]. Other problems associated with problematic substance use include loss of interest in social activities, disorganized thinking, reduced problem-solving performance, and social isolation [2-4,10].

The biopsychosocial model provides a comprehensive explanation of the complex interactions that can occur leading to substance use. This model explains that biological, genetic, personality, psychological, cognitive, social, cultural, and environmental factors interact to produce substance use disorder [14]. Genetic and biological risks include being children of alcohol-dependent parents or the presence of the alcohol dehydrogenase enzyme and dopamine D2A1 gene. For instance, a study examining the association between the gene responsible for alcohol dehydrogenase and alcohol use among the Asian population found that those deficient in this enzyme are less likely to use the substance [15]. However, not all persons with such predispositions develop problems with substance use. A myriad of other psychosocial factors may promote the use of substances, including individual, familial, social, and environmental factors [16]. Factors at the individual level include being a male, LGBTQ+, early exposure to traumatic life events, individuals with a family history of a substance use disorder, prenatal exposure to alcohol and other drugs, sleep problems, and co-occurring psychopathology (e.g., ADHD, depression) $[2,3,17,18]$. Social factors include peer substance use and involvement in romantic relationships at an early age $[19,20]$. Environmental risks include family dysfunction, lack of parental supervision and monitoring, and being street-involved $[2-4,10,11]$. There are also protective factors, including self-efficacy, social competence, academic achievement, personal and social controls against norm-violating behaviors, church attendance, and sense of morality [21-26]. Nurturing home environments with open communication and parental support as well as positive school experience and positive peer role models may also be protective [23,24].

Substance use during a young age might disrupt vital developmental transitions as the brain undergoes cognitive, emotional, and psychosocial development [1]. In addition, this period also coincides with the onset of a variety of mental health conditions [27-30]. Neurodevelopmental research has demonstrated that the brains of youth may be more vulnerable to the effects of substances. Hence, they are at risk of developing patterns of behavior that result in substance abuse (continued use regardless of physical or psychosocial problems or dependence) and substance dependence (physiologic dependence demonstrated by withdrawal symptoms or the development of tolerance to alcohol or other drugs) $[14,31,32]$. These adverse outcomes are particularly concerning for youth who use substances in an attempt to cope with difficult situations such as trauma or victimization due to the dual neurological vulnerability created by traumatic life events and the engagement in substance use [20,23,33-37].

There is a consistent relationship between mental health conditions and problematic substance use among youth $[16-18,23,24]$. Co-occurring mental and substance use conditions are highest among youth aged 15-24 years in Ontario and Canada [2]. Substance use is associated with comorbid illnesses such as depression and bipolar disorders, posttraumatic stress disorder, psychotic symptoms, and disruptive behavioral disorders in both in-patient and outpatient settings $[26,38,39]$. For youth with mental health conditions, early initiation of treatment is significantly associated with a reduced likelihood of developing substance use disorders [26].

Mental health and substance-related service use among young people in Ontario, Canada, has increased over time, with about 1 in 6 receiving needed mental health services [40]. Co-occurrence of substance use also interferes with the treatment of and recovery from mental health disorders, thus requiring specific consideration within the treatment plan and the need for integrated health services [41,42]. Although best practice promotes the integration of mental health and substance use services, evidence suggests this is rarely the case [43]. In Ontario, some historical barriers to integration have included variation in the public funding and administration of youth mental health services and substance use care, as well as variations in the eligibility criteria for services, particularly related to the presence of substance use [43-45]. Consequently, youth have experienced chal- 
lenges accessing and navigating services across multiple service sectors in Ontario [44-47]. There are also substantial delays between the onset of mental health or substance use conditions and access to care, with only the severe cases receiving timely services [48,49]. While recent amendments to public policy have been made to the public administration of child and youth mental health services in Ontario [50], gaps often remain in the integration of mental health and substance use care at the provider level. Youth seen in outpatient settings and those admitted into residential/in-patient settings may demonstrate greater clinical complexity and/or higher rates of substance use compared to youth in the general population.

There is a need to improve the early identification of comorbid mental health conditions and modifiable risk factors associated with substance use. This will help to develop early intervention strategies to potentially reduce the risk of developing substance use disorders and the utilization of high-intensity services. Therefore, this study examines individual factors associated with substance use among youth (12-18 years) accessing services in community-based and residential mental healthcare settings in Ontario, Canada.

\section{Materials and Methods}

\subsection{Methodology}

\subsubsection{Study Population}

The sample comprised 48,118 youth (12-18 years) assessed in the community $(\mathrm{N}=47,418)$ or residential $(\mathrm{N}=700)$ mental health agencies in Ontario, Canada, with either the interRAI Child Youth Mental Health Assessment (interRAI ChYMH) or the interRAI ChYMH Screener (ChYMH-S) between 1 January 2012 data, and 31 October 2020. Referrals were made to the agencies through family physicians, pediatricians, school personnel, parents, or other allied professionals. Data from the first assessment of all youth were selected for the analyses. As part of the license to use the interRAI ChYMH data, sharing agreements are in place for agencies to submit anonymized data to interRAI Canada at the University of Waterloo.

\subsubsection{Instruments}

The interRAI ChYMH is used as part of the standard of care in many agencies and organizations that provide service to children and youth across Ontario. The assessment includes over 400 items to inform treatment planning, including identification information, intake and initial history, mental state indicators, substance use or excessive behavior, harm to self and others, behavior, strengths and resilience, cognition and executive functioning, independence in daily activities, communication and vision, health conditions, family and social relations, stress and trauma, medications, preventions, service utilization and treatments, nutritional status, education, environmental assessment, diagnostic and other health information, service termination and discharge information. Items in the assessment are completed by trained clinicians overseeing the care of the person. Assessors use a semi-structured interview approach that incorporates all available information, including observation, review of the clinical record, and parent report as well as self-report [51]. The ChYMH also includes the interRAI Adolescent Supplement Assessment Form [49]. This supplement to the ChYMH is used when assessing youth aged 13 to 17. It includes additional information on substance use or excessive behavior, independence in daily activities, service utilization, and strengths. The interRAI-ChYMH has demonstrated good psychometric properties in clinical settings [52].

The ChYMH-S is a brief screener that provides an initial assessment for early identification, triaging, and prioritizing services. Taking approximately 15-20 min to complete, the assessment includes the following domains: (1) Mental State Indicators (e.g., Mood disturbance, Anxiety), (2) Substance Use or Excessive Behaviour, (3) Harm to Self and Others, (4) Behaviour, (5) Cognition, Communication and Development, (6) Stress, Trauma, and Social Relationships, and (7) Education. The screener demonstrated strong inter-item reliability on all measured scales and good convergent validity [53]. 
Dependent Variable: The primary variable of interest was the use of any substance, including misuse of medication and alcohol, in the 14 to 30 days prior to assessment. The ChYMH Adolescent Supplement assesses the most recent instance a youth reported using a given substance, including opiates, cannabis, cocaine, stimulants, inhalants, hallucinogens, and intentional misuse of prescription or over-the-counter medication. Use is coded if the person used the substance in the three days, seven days, 30 days, one year, and more than one year before assessment. The ChYMH-S assesses whether the person used substances or intentionally misused prescription or over-the-counter medication in the 14 days before the assessment.

Independent Variables: The interRAI ChYMH and ChYMH-S include a range of clinical, functional, social, and familial variables that were considered in the biopsychosocial model in the examination of factors associated with youth substance use. The biopsychosocial model posits that biological, genetic, personality, psychological, cognitive, social, cultural, and environmental factors interact, leading to the use of substances and substance use disorders [14]. Using this model as a framework, variables in the ChYMH and ChYMH-S could be grouped into demographic factors that include age, sex, education, employment, and insurance coverage. Cultural factors included aboriginal identity and if an interpreter was needed to complete the assessment. Clinical characteristics included psychological, personality, and cognitive factors, and psychosocial categories included developmental needs, housing, income and family-related variables, community, and education engagements (See Tables 1 and A1). However, the psychosocial model of adolescent substance use was preferred because it contains modifiable factors that could be addressed. Therefore, variables for the final model were modifiable factors that are available interRAI assessment instruments.

Table 1. Description of independent variables available from the interRAI ChYMH or ChYMH Screener.

\begin{tabular}{lll}
\hline Categories of Independent Variables & Biopsychosocial Model & Variables \\
\hline Demographic information & Biological/genetic & Age, gender \\
Psychosocial needs & Psychosocial & $\begin{array}{l}\text { residential instability, family functioning, } \\
\text { social support, education engagement }\end{array}$ \\
\hline Environmental factors & Environmental & Home environment \\
\hline Clinical characteristics & Psychological, cognitive, personality & $\begin{array}{l}\text { Mental health symptoms, cognition, } \\
\text { harm to self, behavior, history of trauma } \\
\text { or stress }\end{array}$ \\
\hline Interventions & & $\begin{array}{l}\text { Treatment, control intervention, } \\
\text { adherence to treatment, prevention }\end{array}$ \\
\hline Service use history & & $\begin{array}{l}\text { Community mental health agency, } \\
\text { residential/in-patient, emergency room }\end{array}$ \\
\hline
\end{tabular}

In addition to these items, this study examined three scales embedded in the ChYMH and the ChYMH-S: Anxiety Scale, Distraction/Hyperactive Scale, and Depression Severity Index. The Anxiety Scale is a seven-item scale that assesses the frequency of several anxiety symptoms (i.e., repetitive anxious concerns, unrealistic fears, obsessive thoughts, compulsive behavior, intrusive thoughts or flashbacks, episodes of panic, and nightmares). The total sum of the frequency of each symptom (4-point scale where $0=$ not present to 4 = Exhibited daily in last 3 days, 3 or more episodes or continuously) ranges from 0 to 28. Thus, higher scores indicate higher levels of anxiety [52]. The Cronbach's alpha for the Anxiety Scale was 0.75 in the current sample. Hyperactive/Distraction Scale is a 4-item scale that assesses the frequency of four facets of distractibility and hyperactivity. That is impulsivity, ease of distraction, hyperactivity, and disorganization). The total sum of the frequency of each behavior $(0=$ not present to $4=$ Exhibited daily in the last 3 days, 3 or more episodes or continuously) ranges from 0 to 16 . Higher scores indicated higher levels of 
distractibility and hyperactivity [52]. The Cronbach's alpha for the Hyperactive/Distraction scale was 0.77 in the current sample. The Depression Severity Scale is comprised of five items, capturing various depressive expressions of the individual, including sad or pained facial expressions, made negative statements, self-deprecation, expressions of guilt/shame, and hopelessness. Possible scores for each of the items are 0 ("Not present"), 1 ("Present but not exhibited in last 3 days"), 2 ("Exhibited on 1-2 of last 3 days"), and 3 ("Exhibited daily in last 3 days"). Higher scores indicated higher levels of depressive symptoms [54]. The Cronbach's alpha for the Depression Severity scale was 0.83 in the current sample. The Cronbach's alphas for the three scales in this study sample were more than 0.7 , indicating good internal consistencies.

\subsubsection{Analytic Approach}

Separate analyses were done for the residential and community datasets. Descriptive analysis of the study population performed includes frequency and percentages for categorical data for both settings. Bivariable relationships between the independent variables and substance use were examined using chi-square analyses due to the categorical nature of the data.

Modified Poisson GEE regression model was used to estimate the effect size of the association between the independent variables and substance use for variables with significant bivariable associations to estimate the relative risk for community and residential datasets. Regression models using GEE are considered marginal, or population-averaged, models that account for clustering of observations (i.e., correlation of responses) within organizations by including organization identification number (ID) as a source of random error in each model [55-57]. The GENMOD Procedure in the Statistical Analysis Software version 9.4 was used to assess the risk ratio with a $95 \%$ confidence interval using a REPEAT statement to specify the GEE procedure. The organization ID in the ChYMH dataset was entered as the clustering variable using the Subject option (subject identifier). The independent correlation structure assumes that each individual and individual subject is independent [58].

The variables were entered in blocks into the model (e.g., biological factors, psychosocial factors, environmental factors, and clinical factors). Different combinations of the independent variables were examined to rule out order-of-entry, deletion effects, and multicollinearity $[58,59]$. For inclusion in the final risk model, variables needed to be statistically related to substance use (i.e., parameter estimates with $p$-values less than 0.01) with risk ratios greater than 1.3 or below 0.77 .

Many methods to evaluate the goodness of fit for GEE regression models have been proposed in simulation studies [60]. For instance, the goodness of fit statistics QIC and QICu can compare the models' strengths with lower QIC and QICu values indicating better model performance [61]. However, these methods are not routinely implemented in SAS output, and consensus on the goodness of fit statistic for GEE models has not been established. Therefore, final risk models using GEEs identified in the ChYMH dataset were subjected to logistic regression. Using logistic regression, the model's discriminatory power was evaluated using the c statistic [62]. The c statistic measures how well the model discriminates those who experience an event (e.g., outcome) from those who do not [63]. For example, a c statistic of 0.5 indicates the model is no more discriminating than chance, while a statistic of 1.0 indicates perfect discriminatory power.

\section{Results}

Descriptive and clinical characteristics of the community and residential sample are in Table 2. The community sample was predominantly female $(60.4 \%)$, while just over half of the residential sample were male (53.9\%). About a third of youth in the community sample were victims of physical, sexual, or emotional abuse (36.4\%) compared to $59.0 \%$ within the residential sample. The majority of youth assessed in the residential and community 
settings had contacts with community mental health agencies or professionals prior to assessment, $87 \%$ and $69 \%$, respectively.

Table 2. Characteristics of the study population.

\begin{tabular}{|c|c|c|c|c|c|c|c|c|c|c|c|c|c|}
\hline \multirow[b]{3}{*}{ Characteristics } & \multirow[b]{3}{*}{ Level } & \multicolumn{6}{|c|}{ Community } & \multicolumn{6}{|c|}{ Residential } \\
\hline & & \multicolumn{2}{|c|}{ Total $(\mathrm{N}=47,418)$} & \multicolumn{2}{|c|}{$\mathrm{M}(\mathrm{N}=18,790)$} & \multicolumn{2}{|c|}{$\mathrm{F}(\mathrm{N}=28,628)$} & \multicolumn{2}{|c|}{ Total $(\mathrm{N}=700)$} & \multicolumn{2}{|c|}{$M(N=377)$} & \multicolumn{2}{|c|}{$F(N=323)$} \\
\hline & & $\mathrm{n}$ & $\%$ & $\mathrm{n}$ & $\%$ & $\mathrm{n}$ & $\%$ & $\mathrm{n}$ & $\%$ & $\mathrm{n}$ & $\%$ & $\mathrm{n}$ & $\%$ \\
\hline \multirow{2}{*}{ Instrument } & ChYMH-S & 38,972 & 82.2 & & & & & 4 & 0.6 & & & & \\
\hline & ChYMH & 8446 & 17.8 & & & & & 694 & 99.4 & & & & \\
\hline \multirow{2}{*}{ Sex } & Male & 18,790 & 39.6 & & & & & 377 & 53.9 & & & & \\
\hline & Female & 28,628 & 60.4 & & & & & 323 & 46.1 & & & & \\
\hline \multirow{3}{*}{ Age group } & 12-14 & 13,623 & 28.7 & 6350 & 33.8 & 7273 & 25.4 & 178 & 25.4 & 107 & 28.4 & 71 & 22 \\
\hline & $15-16$ & 18,149 & 38.3 & 6782 & 36.1 & 11,367 & 39.7 & 302 & 43.1 & 158 & 41.9 & 144 & 44.6 \\
\hline & $17-18$ & 15,646 & 33 & 5658 & 30.1 & 9988 & 34.9 & 220 & 31.4 & 11 & 29.7 & 108 & 33.4 \\
\hline \multirow{3}{*}{$\begin{array}{l}\text { Risk of disrupted } \\
\text { education }\end{array}$} & Yes & 27,580 & 58.6 & 11,859 & 63.6 & 15,721 & 55.2 & 417 & 61.6 & 236 & 65 & 181 & 57.6 \\
\hline & No & 18,934 & 40.2 & 6503 & 34.9 & 12,431 & 43.7 & 238 & 35.2 & 111 & 30.6 & 127 & 40.5 \\
\hline & $\begin{array}{l}\text { Not } \\
\text { applicable }\end{array}$ & 582 & 1.2 & 277 & 1.5 & 305 & 1.1 & 22 & 3.2 & 16 & 4.4 & 6 & 1.9 \\
\hline \multirow{2}{*}{$\begin{array}{l}\text { No Strong and } \\
\text { supportive } \\
\text { relationship with } \\
\text { family }\end{array}$} & Yes & 8542 & 18 & 2958 & 15.7 & 5584 & 19.5 & 238 & 34 & 107 & 28.4 & 131 & 40.6 \\
\hline & No & 38,876 & 82 & 15,832 & 84.3 & 23,044 & 80.5 & 462 & 66 & 270 & 71.6 & 192 & 59.4 \\
\hline \multirow{2}{*}{$\begin{array}{l}\text { Parent/caregiver } \\
\text { has mental health } \\
\text { issue }\end{array}$} & Yes & 17,150 & 36.2 & 6423 & 34.2 & 10,727 & 37.5 & 349 & 49.9 & 175 & 46.4 & 174 & 53.9 \\
\hline & No & 30,268 & 63.8 & 12,367 & 65.8 & 17,901 & 62.5 & 351 & 50.1 & 202 & 53.6 & 149 & 46.1 \\
\hline \multirow{3}{*}{$\begin{array}{l}\text { Parental addic- } \\
\text { tion/substance } \\
\text { use }\end{array}$} & Yes & 7537 & 20.1 & 3531 & 18.8 & 6006 & 21 & 279 & 39.9 & 153 & 40.6 & 126 & 39 \\
\hline & No & 37,881 & 79.9 & 15,259 & 81.2 & 22,622 & 79 & 421 & 60.1 & 224 & 59.4 & 197 & 61 \\
\hline & $\geq 5$ & 1719 & 3.6 & 681 & 3.6 & 1038 & 3.6 & 43 & 6.1 & 23 & 6.1 & 20 & 6.2 \\
\hline \multirow{2}{*}{$\begin{array}{l}\text { Use of any } \\
\text { substance in the } \\
\text { last 14-30 days }\end{array}$} & Yes & 10,587 & 22.3 & 4150 & 22.1 & 6437 & 22.5 & 259 & 37 & 139 & 36.9 & 120 & 37.1 \\
\hline & No & 36,831 & 77.7 & 14,640 & 77.9 & 22,191 & 77.5 & 441 & 63 & 238 & 63.1 & 203 & 62.9 \\
\hline \multirow{2}{*}{ Behavior symptom } & Yes & 26,014 & 54.9 & 11,821 & 62.9 & 14,193 & 49.6 & 579 & 82.7 & 332 & 88.1 & 247 & 76.5 \\
\hline & No & 21,404 & 45.1 & 6969 & 37.1 & 14,435 & 50.4 & 121 & 17.3 & 45 & 11.9 & 76 & 23.5 \\
\hline \multirow{2}{*}{$\begin{array}{l}\text { Self-injurious } \\
\text { ideation or attempt }\end{array}$} & Yes & 27,605 & 58.2 & 8217 & 43.7 & 19,388 & 67.7 & 458 & 65.4 & 201 & 53.3 & 257 & 79.6 \\
\hline & No & 19,813 & 41.8 & 10,573 & 56.3 & 9240 & 32.3 & 242 & 34.6 & 176 & 46.7 & 66 & 20.4 \\
\hline \multirow{2}{*}{$\begin{array}{l}\text { Problematic sexual } \\
\text { behavior }\end{array}$} & Yes & 2683 & 5.7 & 954 & 5.1 & 1729 & 6 & 74 & 10.6 & 39 & 10.3 & 35 & 10.8 \\
\hline & No & 44,735 & 94.3 & 17,836 & 94.9 & 26,899 & 94 & 626 & 89.4 & 338 & 89.7 & 288 & 89.2 \\
\hline \multirow{2}{*}{ Victim of abuse } & Yes & 17,247 & 36.4 & 5505 & 29.3 & 11,742 & 41 & 413 & 59 & 208 & 55.2 & 205 & 63.5 \\
\hline & No & 30,171 & 73.6 & 13,285 & 70.7 & 16,886 & 59 & 287 & 41 & 169 & 44.8 & 118 & 36.5 \\
\hline Distraction and & 0-None & 9550 & 20.1 & 3056 & 16.3 & 6494 & 22.7 & 82 & 11.7 & 34 & 9 & 48 & 14.9 \\
\hline hyperactivity scale & 1-Low & 28,063 & 59.1 & 10,692 & 56.9 & 17,371 & 60.7 & 403 & 57.6 & 207 & 54.9 & 196 & 60.7 \\
\hline & 2-Moderate & 3719 & 7.8 & 1685 & 9 & 2034 & 7.1 & 61 & 8.7 & 34 & 9 & 27 & 8.4 \\
\hline & 3-High & 3023 & 6.4 & 1570 & 8.4 & 1453 & 5.1 & 78 & 11.4 & 56 & 14.9 & 22 & 6.8 \\
\hline & 4-Very high & 3063 & 6.5 & 1787 & 9.5 & 1276 & 4.4 & 76 & 10.9 & 46 & 12.2 & 30 & 9.3 \\
\hline & 0-None & 8145 & 17.2 & 4484 & 23.9 & 3661 & 12.8 & 160 & 22.9 & 99 & 26.3 & 61 & 18.9 \\
\hline & 1-Low & 13,262 & 28 & 5751 & 30.6 & 7511 & 26.2 & 191 & 27.3 & 109 & 28.9 & 82 & 25.4 \\
\hline Anxiety scale & 2-Moderate & 16,275 & 34.3 & 5844 & 31.1 & 10,431 & 36.4 & 204 & 29.1 & 107 & 28.4 & 97 & 30 \\
\hline & 3-High & 7839 & 16.5 & 2269 & 12.1 & 5570 & 19.4 & 105 & 15 & 54 & 14.3 & 51 & 15.8 \\
\hline & 4-Very high & 1897 & 4 & 442 & 2.3 & 1455 & 5.1 & 40 & 5.7 & 8 & 2.1 & 32 & 9.9 \\
\hline
\end{tabular}


Table 2. Cont.

\begin{tabular}{|c|c|c|c|c|c|c|c|c|c|c|c|c|c|}
\hline \multirow[b]{3}{*}{ Characteristics } & \multirow[b]{3}{*}{ Level } & \multicolumn{6}{|c|}{ Community } & \multicolumn{6}{|c|}{ Residential } \\
\hline & & \multicolumn{2}{|c|}{ Total $(\mathrm{N}=47,418)$} & \multicolumn{2}{|c|}{$M(N=18,790)$} & \multicolumn{2}{|c|}{$\mathrm{F}(\mathrm{N}=28,628)$} & \multicolumn{2}{|c|}{ Total $(\mathrm{N}=700)$} & \multicolumn{2}{|c|}{$M(N=377)$} & \multicolumn{2}{|c|}{$F(N=323)$} \\
\hline & & $\mathrm{n}$ & $\%$ & $\mathrm{n}$ & $\%$ & $\mathrm{n}$ & $\%$ & $\mathrm{n}$ & $\%$ & $\mathrm{n}$ & $\%$ & $\mathrm{n}$ & $\%$ \\
\hline \multirow{5}{*}{$\begin{array}{l}\text { Depression } \\
\text { severity Index }\end{array}$} & 0-None & 1921 & 4.1 & 993 & 5.3 & 928 & 3.2 & 40 & 5.7 & 24 & 6.4 & 16 & 5 \\
\hline & 1-Low & 20,186 & 42.6 & 9362 & 49.8 & 10,824 & 37.8 & 304 & 43.4 & 191 & 50.7 & 113 & 35 \\
\hline & 2-Moderate & 12,675 & 26.7 & 4828 & 25.7 & 7847 & 27.4 & 176 & 25.1 & 89 & 23.6 & 87 & 26.9 \\
\hline & 3-High & 5356 & 11.3 & 1708 & 9.1 & 3648 & 12.7 & 67 & 9.6 & 33 & 8.7 & 34 & 10.5 \\
\hline & 4-Very high & 7280 & 15.3 & 1899 & 10.1 & 5381 & 18.8 & 113 & 16.2 & 40 & 10.6 & 73 & 22.6 \\
\hline \multirow{2}{*}{ Sleep problem } & Yes & 29,868 & 63 & 10,853 & 57.8 & 19,015 & 66.4 & 415 & 59.3 & 222 & 58.9 & 193 & 59.8 \\
\hline & No & 17,550 & 37 & 7937 & 42.2 & 9613 & 33.6 & 285 & 40.7 & 115 & 41.1 & 130 & 40.2 \\
\hline \multirow{2}{*}{ Cognitive problem } & Yes & 8254 & 17.4 & 4429 & 23.6 & 3825 & 13.4 & 326 & 46.6 & 207 & 54.9 & 119 & 36.8 \\
\hline & No & 39,164 & 82.6 & 14,361 & 76.4 & 24,803 & 86.6 & 374 & 53.4 & 170 & 45.1 & 204 & 63.2 \\
\hline \multirow{3}{*}{$\begin{array}{l}\text { Last contact with } \\
\mathrm{CMH} \text { agency in the } \\
\text { last year }\end{array}$} & No contact & 14,973 & 31.6 & 6600 & 35.1 & 8373 & 29.2 & 91 & 13.2 & 47 & 12.7 & 44 & 13.7 \\
\hline & $\geq 31$ & 10,897 & 23 & 4435 & 23.6 & 6462 & 22.6 & 153 & 22.1 & 85 & 23 & 68 & 21.2 \\
\hline & $\leq 30$ & 21,547 & 45.4 & 7754 & 41.3 & 13,793 & 48.2 & 447 & 64.7 & 238 & 64.3 & 209 & 65.1 \\
\hline
\end{tabular}

There were several differences in clinical characteristics among males and females in the community agencies. For instance, a larger proportion of females reported selfinjurious ideation $(67.7 \%)$, anxiety $(87.1 \%)$, and abuse $(41.0 \%)$, compared to males $(43.7 \%$, $76.1 \%$, and $29.3 \%$, respectively). Alternatively, a higher proportion of males experienced behavioral symptoms $(62.9 \%)$ and cognitive problems $(23.6 \%)$ compared to females $(49.6 \%$ and $13.4 \%$, respectively). In the residential settings, a higher proportion of females reported a lack of strong family support $(40.6 \%)$, having a parent with $\mathrm{MH}$ issues $(53.9 \%)$, history of self-injurious ideation/attempt (79.6\%), abuse (63.5\%) and anxiety $(81.1 \%)$ compared to males $(28.4 \%, 46.4 \%, 6.4 \%, 53.3 \%, 55.2 \%$, and $73.7 \%$ respectively). More males experienced cognitive problems $(54.9 \%)$, and HDS $(91.0 \%)$ compared to females $(26.0 \%, 36.8 \%$, and $85.2 \%$, respectively). The proportion of youth who reported any substance use in the 14-30 days before the assessment was larger in the residential settings (37.1\% females and $36.9 \%$ males) than in the community setting (22.1.0\% females and $22.5 \%$ males).

Bivariable analyses of factors associated with substance use in the 14-30 days prior to assessment for the community sample are shown in Tables 3 and 4 for the residential sample. While most variables had a significant bivariable association in community settings, variables associated with substance use in residential settings were limited to age, self-injurious ideation/attempt, behavioral symptoms, cognitive problem, being a victim of abuse, being at risk of disrupted education, lack of family support, and having parent/caregiver with SUD. There was no statistically significant difference in the proportion of males compared to females that have used substances in the last 14-30 days in the community (bivariate $\mathrm{OR}=1.02,95 \%$ CI: $[0.98,1.07] ; p$-value $=0.31$ ) and residential (bivariable $\mathrm{OR}=1.01,95 \% \mathrm{CI}$ : $[0.74,1.38] ; p$-value $=0.94)$ settings. However, the proportion of youth who used substances significantly increased as youth age increased. In the community setting, the proportion of youth who used substances increased with age from $5.0 \%$ of youth aged $12-14$ to $36.8 \%$ of youth aged 17-18. In the residential setting, the proportion of youth who used substances increased from $16.0 \%$ among youth aged $12-14$ to $49.6 \%$ of youth aged $17-18$. This pattern of substance use across age categories was similar among males and females in both community and residential settings. However, the proportion increased more by age for females compared to males. 
Table 3. The proportion of youth using substances in community settings, by gender.

\begin{tabular}{|c|c|c|c|c|c|c|c|c|c|c|c|c|c|}
\hline \multirow[b]{2}{*}{ Characteristics } & \multirow[b]{2}{*}{ Level } & \multicolumn{4}{|c|}{ Total Community Sample } & \multicolumn{4}{|c|}{ Males } & \multicolumn{4}{|c|}{ Females } \\
\hline & & $\mathbf{n}$ & $\%$ & $X^{2}$ & $p$-Value & n & $\%$ & $\mathrm{X}^{2}$ & $p$-Value & $\mathbf{n}$ & $\%$ & $X^{2}$ & $p$-Value \\
\hline \multirow[t]{2}{*}{ Sex } & Male & 4150 & 22.1 & 1.04 & 0.308 & & & & & & & & \\
\hline & Female & 6437 & 22.5 & & & & & & & & & & \\
\hline \multirow[t]{3}{*}{ Age group } & $12-14$ & 683 & 5.0 & 4255.86 & $<0.0001$ & 263 & 4.1 & 2205.8 & $<0.0001$ & 420 & 5.8 & 2111.85 & $<0.0001$ \\
\hline & $15-16$ & 4141 & 22.8 & & & 1652 & 24.4 & & & 2489 & 21.9 & & \\
\hline & $17-18$ & 5763 & 36.8 & & & 2235 & 39.5 & & & 3528 & 35.3 & & \\
\hline \multirow[t]{4}{*}{$\begin{array}{l}\text { Contact with } \\
\text { CMH }\end{array}$} & $\begin{array}{l}\text { No contact } \\
\text { in the last } \\
\text { year }\end{array}$ & 2823 & 18.9 & 172.06 & $<0.0001$ & 1400 & 21.2 & 9.2 & 0.01 & 1423 & 17 & 218.37 & $<0.0001$ \\
\hline & $31+$ days & 2450 & 22.5 & & & 953 & 21.5 & & & 1497 & 23.2 & & \\
\hline & $\begin{array}{l}30 \text { days or } \\
\text { less }\end{array}$ & 5314 & 24.7 & & & 1797 & 23.2 & & & 3517 & 25.5 & & \\
\hline & 0-None & 1305 & 13.7 & 597.13 & $<0.0001$ & 462 & 15.1 & 117.97 & $<0.0001$ & 843 & 13 & 546.98 & $<0.0001$ \\
\hline \multirow{4}{*}{$\begin{array}{l}\text { Distraction } \\
\text { and } \\
\text { hyperactivity } \\
\text { scale }\end{array}$} & 1-Low & 6614 & 23.6 & & & 2487 & 23.3 & & & 4127 & 23.8 & & \\
\hline & 2-Moderate & 1104 & 29.7 & & & 445 & 26.4 & & & 659 & 32.4 & & \\
\hline & 3-High & 805 & 26.6 & & & 379 & 24.1 & & & 426 & 29.3 & & \\
\hline & 4-Very high & 759 & 24.8 & & & 377 & 21.1 & & & 382 & 29.9 & & \\
\hline \multirow[t]{5}{*}{ Anxiety Scale } & 0-None & 1753 & 21.5 & 134.17 & $<0.0001$ & 1052 & 23.5 & 10.56 & 0.032 & 701 & 19.2 & 187.13 & $<0.0001$ \\
\hline & 1-Low & 2707 & 20.4 & & & 1243 & 21.6 & & & 1464 & 19.5 & & \\
\hline & 2-Moderate & 3573 & 22.0 & & & 1255 & 21.5 & & & 2318 & 22.2 & & \\
\hline & 3-High & 1991 & 25.4 & & & 487 & 21.5 & & & 1504 & 27 & & \\
\hline & 4-Very high & 563 & 29.7 & & & 113 & 25.6 & & & 450 & 30.9 & & \\
\hline \multirow[t]{5}{*}{$\begin{array}{l}\text { Depression } \\
\text { severity Index }\end{array}$} & 0-None & 317 & 16.5 & 758.76 & $<0.0001$ & 188 & 18.9 & 161.48 & $<0.0001$ & 129 & 13.9 & 637.69 & $<0.0001$ \\
\hline & 1-Low & 3623 & 18.0 & & & 1831 & 19.6 & & & 1792 & 16.6 & & \\
\hline & 2-Moderate & 2852 & 22.5 & & & 1064 & 22 & & & 1788 & 22.8 & & \\
\hline & 3-High & 1422 & 26.6 & & & 483 & 28.3 & & & 939 & 25.7 & & \\
\hline & 4-Very high & 2373 & 32.6 & & & 584 & 30.8 & & & 1789 & 33.3 & & \\
\hline \multirow[t]{2}{*}{ Sleep problem } & No & 3300 & 18.8 & 199.47 & $<0.0001$ & 1527 & 19.2 & 64.73 & $<0.0001$ & 1773 & 18.4 & 135.61 & $<0.0001$ \\
\hline & Yes & 7287 & 24.4 & & & 2623 & 24.2 & & & 4664 & 24.5 & & \\
\hline \multirow[t]{2}{*}{$\begin{array}{l}\text { Self-injurious } \\
\text { ideation or } \\
\text { attempt }\end{array}$} & No & 2865 & 14.5 & 1214.5 & $<0.0001$ & 1868 & 17.7 & 274.31 & $<0.0001$ & 997 & 10.8 & 1070.65 & $<0.0001$ \\
\hline & Yes & 7722 & 28.0 & & & 2282 & 27.8 & & & 5440 & 28.1 & & \\
\hline \multirow[t]{2}{*}{$\begin{array}{l}\text { Behavior } \\
\text { symptom }\end{array}$} & No & 3690 & 17.2 & 614.59 & $<0.0001$ & 1250 & 17.9 & 110.85 & $<0.0001$ & 2440 & 16.9 & 520.45 & $<0.0001$ \\
\hline & Yes & 6897 & 26.5 & & & 2900 & 24.5 & & & 3997 & 28.2 & & \\
\hline \multirow[t]{2}{*}{$\begin{array}{l}\text { Problematic } \\
\text { sexual } \\
\text { behavior }\end{array}$} & No & 7547 & 21.3 & 442.98 & $<0.0001$ & 3892 & 21.8 & 14.36 & 0.0002 & 5655 & 21 & 546.12 & $<0.0001$ \\
\hline & Yes & 1040 & 38.8 & & & 258 & 27 & & & 782 & 45.2 & & \\
\hline \multirow[t]{2}{*}{$\begin{array}{l}\text { Cognitive } \\
\text { problem }\end{array}$} & No & 9299 & 23.7 & 260.42 & $<0.0001$ & 3530 & 24.6 & 220.27 & $<0.0001$ & 5769 & 23.3 & 63.86 & $<0.0001$ \\
\hline & Yes & 1288 & 15.6 & & & 620 & 14 & & & 668 & 17.5 & & \\
\hline \multirow[t]{2}{*}{$\begin{array}{l}\text { Victim of } \\
\text { abuse }\end{array}$} & No & 5073 & 16.8 & 1453.67 & $<0.0001$ & 2499 & 18.8 & 282.72 & $<0.0001$ & 2574 & 15.2 & 1238.69 & $<0.0001$ \\
\hline & Yes & 5514 & 32.0 & & & 1651 & 30 & & & 3863 & 32.9 & & \\
\hline \multirow[t]{2}{*}{$\begin{array}{l}\text { No Strong } \\
\text { family } \\
\text { relationship }\end{array}$} & No & 7577 & 19.5 & 1001.43 & $<0.0001$ & 3067 & 19.4 & 430.49 & $<0.0001$ & 4510 & 19.6 & 575.47 & $<0.0001$ \\
\hline & Yes & 3010 & 35.2 & & & 1083 & 36.6 & & & 1927 & 34.5 & & \\
\hline
\end{tabular}


Table 3. Cont.

\begin{tabular}{|c|c|c|c|c|c|c|c|c|c|c|c|c|c|}
\hline \multirow[b]{2}{*}{ Characteristics } & \multirow[b]{2}{*}{ Level } & \multicolumn{4}{|c|}{ Total Community Sample } & \multicolumn{4}{|c|}{ Males } & \multicolumn{4}{|c|}{ Females } \\
\hline & & $\mathrm{n}$ & $\%$ & $X^{2}$ & $p$-Value & $\mathrm{n}$ & $\%$ & $X^{2}$ & $p$-Value & $\mathbf{n}$ & $\%$ & $X^{2}$ & $p$-Value \\
\hline \multirow[t]{2}{*}{$\begin{array}{l}\text { Parental } \\
\text { addic- } \\
\text { tion/substance } \\
\text { abuse }\end{array}$} & No & 7207 & 19.0 & 1183.86 & $<0.0001$ & 2933 & 19.2 & 387.26 & $<0.0001$ & 4274 & 18.9 & 798.18 & $<0.0001$ \\
\hline & Yes & 3380 & 35.4 & & & 1217 & 34.5 & & & 2163 & 36 & & \\
\hline \multirow[t]{2}{*}{$\begin{array}{l}\text { Parent with } \\
\text { MH issue }\end{array}$} & No & 6253 & 20.7 & 134.29 & $<0.0001$ & 2655 & 21.5 & 8.02 & 0.005 & 3598 & 20.1 & & \\
\hline & Yes & 4334 & 25.3 & & & 1495 & 23.3 & & & 2839 & 26.5 & & \\
\hline \multirow[t]{3}{*}{$\begin{array}{l}\text { Risk of } \\
\text { disrupted } \\
\text { education }\end{array}$} & No & 2795 & 14.8 & 1042.04 & $<0.0001$ & 991 & 15.2 & 270.16 & $<0.0001$ & 1804 & 14.5 & 805.99 & $<0.0001$ \\
\hline & Yes & 7545 & 27.4 & & & 3038 & 25.6 & & & 4507 & 28.7 & & \\
\hline & NA & 168 & 28.9 & & & 79 & 28.5 & & & 89 & 29.2 & & \\
\hline
\end{tabular}

Table 4. Pattern of substance use among youth with mental health conditions assessed in residential settings stratified by sex.

\begin{tabular}{|c|c|c|c|c|c|c|c|c|c|c|c|c|c|}
\hline & & \multicolumn{4}{|c|}{ Total Residential Sample } & \multicolumn{4}{|c|}{ Male (N = 377) } & \multicolumn{4}{|c|}{ Female $(\mathrm{N}=323)$} \\
\hline & & $\mathrm{n}$ & $\%$ & $\mathrm{X}^{2}$ & $p$-Value & $\mathbf{n}$ & $\%$ & $\mathrm{X}^{2}$ & $p$-Value & $\mathbf{n}$ & $\%$ & $\mathrm{X}^{2}$ & $p$-Value \\
\hline \multirow[t]{2}{*}{ Sex } & Male & 139 & 36.9 & 0.006 & 0.94 & & & & & & & & \\
\hline & Female & 120 & 37.2 & & & & & & & & & & \\
\hline \multirow[t]{3}{*}{ Age group } & $12-14$ & 29 & 16.3 & 48.82 & $<0.0001$ & 20 & 18.7 & 24.1 & $<0.0001$ & 9 & 12.7 & 25.39 & $<0.0001$ \\
\hline & $15-16$ & 121 & 40.1 & & & 63 & 39.9 & & & 58 & 40.3 & & \\
\hline & $17-18$ & 109 & 49.6 & & & 56 & 50 & & & 53 & 49.1 & & \\
\hline \multirow{3}{*}{$\begin{array}{l}\text { Contact with } \\
\text { Community } \\
\text { Mental Health } \\
\text { in Prior Year }\end{array}$} & No contact & 31 & 34.1 & 2.09 & 0.35 & 24 & 51.1 & 5.06 & 0.08 & 7 & 15.9 & 11.21 & 0.004 \\
\hline & $31+$ days & 51 & 33.3 & & & 27 & 31.8 & & & 24 & 35.3 & & \\
\hline & $\begin{array}{l}30 \text { days or } \\
\text { less }\end{array}$ & 175 & 39.2 & & & 86 & 36.1 & & & 89 & 42.6 & & \\
\hline \multirow{5}{*}{$\begin{array}{l}\text { Distraction } \\
\text { and } \\
\text { hyperactivity } \\
\text { scale }\end{array}$} & 0-None & 30 & 36.6 & 3.15 & 0.53 & 13 & 38.2 & 6.35 & 0.18 & 17 & 35.4 & 5.75 & 0.22 \\
\hline & 1-Low & 152 & 37.7 & & & 86 & 41.6 & & & 66 & 33.7 & & \\
\hline & 2-Moderate & 27 & 44.3 & & & 12 & 35.3 & & & 15 & 55.6 & & \\
\hline & 3-High & 27 & 34.6 & & & 17 & 30.4 & & & 10 & 45.5 & & \\
\hline & 4-Very high & 23 & 30.3 & & & 11 & 23.9 & & & 12 & 40 & & \\
\hline \multirow[t]{5}{*}{ Anxiety Scale } & 0-None & 77 & 48.1 & 11.83 & 0.02 & 56 & 56.6 & 23 & 0.0001 & 21 & 34.4 & 4.07 & 0.4 \\
\hline & 1-Low & 61 & 31.9 & & & 33 & 30.3 & & & 28 & 34.2 & & \\
\hline & 2-Moderate & 69 & 33.8 & & & 33 & 30.8 & & & 36 & 37.1 & & \\
\hline & 3-High & 39 & 37.1 & & & 14 & 25.9 & & & 25 & 49 & & \\
\hline & 4-Very high & 13 & 32.5 & & & $*$ & & & & 10 & 31.3 & & \\
\hline \multirow[t]{5}{*}{$\begin{array}{l}\text { Depression } \\
\text { severity Index }\end{array}$} & 0-None & 16 & 40 & 1.15 & 0.89 & 11 & 45.8 & 5.02 & 0.29 & 5 & 31.3 & 4.52 & 0.34 \\
\hline & 1-Low & 111 & 36.5 & & & 76 & 39.8 & & & 35 & 31 & & \\
\hline & 2-Moderate & 65 & 36.9 & & & 26 & 29.2 & & & 39 & 44.8 & & \\
\hline & 3-High & 28 & 41.8 & & & 14 & 42.4 & & & 14 & 41.2 & & \\
\hline & 4-Very high & 39 & 34.5 & & & 12 & 30 & & & 27 & 37 & & \\
\hline \multirow[t]{2}{*}{ Sleep problem } & No & 95 & 33.3 & 2.77 & 0.1 & 55 & 35.5 & 0.2 & 0.64 & 40 & 30.8 & 3.8 & 0.05 \\
\hline & Yes & 164 & 39.5 & & & 84 & 37.8 & & & 80 & 41.5 & & \\
\hline \multirow{2}{*}{$\begin{array}{l}\text { Self-injurious } \\
\text { ideation or } \\
\text { attempt }\end{array}$} & No & 64 & 26.5 & 17.67 & $<0.0001$ & 58 & 33 & 2.17 & 0.14 & 6 & 9.1 & 27.97 & $<0.0001$ \\
\hline & Yes & 195 & 42.6 & & & 81 & 40.3 & & & 114 & 44.4 & & \\
\hline \multirow[t]{2}{*}{$\begin{array}{l}\text { Behaviour } \\
\text { symptom }\end{array}$} & No & 32 & 26.5 & 6.99 & 0.008 & 15 & 33.3 & 0.27 & 0.6 & 17 & 22.4 & 9.3 & 0.002 \\
\hline & Yes & 227 & 39.2 & & & 124 & 37.4 & & & 103 & 41.7 & & \\
\hline
\end{tabular}


Table 4. Cont.

\begin{tabular}{|c|c|c|c|c|c|c|c|c|c|c|c|c|c|}
\hline & & \multicolumn{4}{|c|}{ Total Residential Sample } & \multicolumn{4}{|c|}{ Male $(\mathrm{N}=377)$} & \multicolumn{4}{|c|}{ Female $(\mathrm{N}=323)$} \\
\hline & & n & $\%$ & $\mathrm{X}^{2}$ & $p$-Value & $\mathbf{n}$ & $\%$ & $\mathrm{X}^{2}$ & $p$-Value & n & $\%$ & $X^{2}$ & $p$-Value \\
\hline \multirow[t]{2}{*}{$\begin{array}{l}\text { Problematic } \\
\text { sexual } \\
\text { behaviour }\end{array}$} & No & 231 & 36.9 & 0.03 & 0.88 & 130 & 38.5 & 3.56 & 0.06 & 101 & 35.1 & 4.93 & 0.03 \\
\hline & Yes & 28 & 37.8 & & & 9 & 23.1 & & & 19 & 54.3 & & \\
\hline \multirow[t]{2}{*}{$\begin{array}{l}\text { Cognitive } \\
\text { problem }\end{array}$} & No & 170 & 45.5 & 24.63 & $<0.0001$ & 85 & 50 & 22.93 & $<0.0001$ & 85 & 41.7 & 4.83 & 0.028 \\
\hline & Yes & 89 & 27.3 & & & 54 & 26.1 & & & 35 & 29.4 & & \\
\hline \multirow[t]{2}{*}{$\begin{array}{l}\text { Victim of } \\
\text { abuse }\end{array}$} & No & 68 & 23.7 & 36.95 & $<0.0001$ & 43 & 25.4 & 17.18 & $<0.0001$ & 25 & 21.1 & 20.3 & $<0.0001$ \\
\hline & Yes & 191 & 46.3 & & & 96 & 46.15 & & & 95 & 46.3 & & \\
\hline \multirow[t]{2}{*}{$\begin{array}{l}\text { No Strong } \\
\text { family } \\
\text { relationship }\end{array}$} & No & 147 & 31.8 & 15.65 & $<0.0001$ & 87 & 32.2 & 8.83 & 0.003 & 60 & 31.3 & 7.06 & 0.008 \\
\hline & Yes & 112 & 47.1 & & & 52 & 48.6 & & & 60 & 45.8 & & \\
\hline \multirow[t]{2}{*}{$\begin{array}{l}\text { Parental } \\
\text { addic- } \\
\text { tion/substance } \\
\text { abuse }\end{array}$} & No & 122 & 29 & 29.16 & $<0.0001$ & 62 & 27.7 & 20.03 & $<0.0001$ & 60 & 30.5 & 9.69 & 0.002 \\
\hline & Yes & 137 & 49.1 & & & 77 & 50.3 & & & 60 & 47.6 & & \\
\hline \multirow[t]{2}{*}{$\begin{array}{l}\text { Parent with } \\
\text { MH issue }\end{array}$} & No & 130 & 37 & 0 & 0.98 & 76 & 37.6 & 0.11 & 0.745 & 54 & 36.2 & 0.1 & 0.75 \\
\hline & Yes & 129 & 37 & & & 63 & 36 & & & 66 & 37.9 & & \\
\hline \multirow[t]{3}{*}{$\begin{array}{l}\text { Risk of } \\
\text { disrupted } \\
\text { education }\end{array}$} & No & 55 & 23.1 & 28.18 & $<0.0001$ & 22 & 19.8 & 18.68 & $<0.0001$ & 33 & 26 & 10.5 & 0.005 \\
\hline & Yes & 180 & 43.2 & & & 101 & 42.8 & & & 79 & 43.7 & & \\
\hline & NA & 11 & 50 & & & 8 & 50 & & & * & * & & \\
\hline
\end{tabular}

Factors Associated with Substance Use among Youth Assessed in Outpatient and In-Patient Mental Health Agencies in Ontario

Table 5 shows the results of the GEE models showing factors associated with substance use among the total samples and by gender in community and residential mental health settings. Only variables that were statistically significant are shown in the models. Among youth assessed in the community $(\mathrm{N}=47,418)$, holding all other variables constant, the relative risk of substance use is significantly lower among females compared to males $(\mathrm{RR}=0.87,95 \% \mathrm{CI}:[0.82,0.93])$. Contact with mental health agencies, sleep problems, DSI, and having parents/caregivers with mental health issues were not significantly associated with substance use in the community sample. Factors associated with a decreased relative risk of substance use include being female, having anxiety symptoms (ANX Scale), and cognition problems were associated with reduced risk of substance use in the community sample.

In residential/inpatient settings $(\mathrm{N}=700)$, similar factors to those in the community sample were associated with substance use (See Table 5). However, several factors not associated in the residential setting include being female ( $R R=0.91,95 \% \mathrm{CI}:[0.71,1.16]$ ), parent mental health issue ( $R R=0.92 ; 95 \% \mathrm{CI}$ : $[0.76,1.12])$, no strong family relationship $(\mathrm{RR}=1.11,95 \% \mathrm{CI}:[0.93,1.33])$, behavioral symptoms $(\mathrm{RR}=1.33,95 \% \mathrm{CI}:[0.98,1.82])$, problematic sexual behavior $(\mathrm{RR}=1.11,95 \% \mathrm{CI}$ : $[0.75,1.64])$, sleep problem $(\mathrm{RR}=1.15,95 \%$ CI: $[0.93,1.42])$, DSI, hyperactivity and distractibility symptoms and anxiety scales include 1:00 (no difference in risk). 
Table 5. Multivariable logistic regression using GEE examining factors associated with substance use among the community and residential settings by gender.

\begin{tabular}{|c|c|c|c|c|}
\hline \multicolumn{5}{|c|}{ Multivariable Regression Analysis (GEE Model) } \\
\hline Community & & & Residential & \\
\hline Total Sample Model & Male Model & Female Model & Total Sample Model & \\
\hline Variables & $\mathrm{RR}(95 \% \mathrm{CI})$ & RR $(95 \%$ CI $)$ & $\mathrm{RR}(95 \% \mathrm{CI})$ & $\mathrm{RR}(95 \% \mathrm{CI})$ \\
\hline $\operatorname{Sex}(\operatorname{Ref}=\mathrm{M})$ & $0.88(0.28,0.94)$ & & & \\
\hline \multicolumn{5}{|l|}{ Age Category } \\
\hline \multicolumn{5}{|l|}{ 12-14 (Reference) } \\
\hline $15-16$ & $4.19(3.61,4.85)$ & $5.63(4.81,6.59)$ & $3.28(2.81,3.84)$ & $2.11(1.56,2.85)$ \\
\hline $17-18$ & $6.50(5.53,7.64)$ & $8.96(7.65,10.5)$ & $5.03(4.23,5.99)$ & $2.52(1.73,3.65)$ \\
\hline \multicolumn{5}{|l|}{$\begin{array}{l}\text { Risk of disrupted } \\
\text { education: }\end{array}$} \\
\hline \multicolumn{5}{|l|}{ None (Reference) } \\
\hline Yes & $1.46(1.35,1.57)$ & $1.39(1.24,1.56)$ & $1.48(1.39,1.57)$ & $1.89(1.37,2.61)$ \\
\hline Not Applicable & $1.35(1.15,1.60)$ & $1.31(1.07,1.61)$ & $1.35(1.11,1.64)$ & $1.88(1.22,2.90)$ \\
\hline \multicolumn{5}{|l|}{$\begin{array}{l}\text { Hyperactivity and } \\
\text { Distractibility Scale }\end{array}$} \\
\hline \multicolumn{5}{|l|}{ None (Ref) } \\
\hline Low & $1.38(1.26,1.50)$ & $1.37(1.23,1.51)$ & $1.35(1.23,1.49)$ & \\
\hline Moderate & $1.58(1.40,1.79)$ & $1.61(1.39,1.87)$ & $1.53(1.35,1.74)$ & \\
\hline High & $1.57(1.39,1.77)$ & $1.64(1.43,1.88)$ & $1.49(1.30,1.70)$ & \\
\hline Very high & $1.59(1.42,1.78)$ & $1.64(1.42,1.90)$ & $1.55(1.34,1.78)$ & \\
\hline \multicolumn{5}{|l|}{ Anxiety: None (Ref) } \\
\hline ANX: Low & $0.82(0.78,0.88)$ & $0.83(0.78,0.89)$ & $0.85(0.79,0.92)$ & $0.60(0.45,0.80)$ \\
\hline ANX: Moderate & $0.75(0.69,0.82)$ & $0.75(0.69,0.83)$ & $0.78(0.71,0.86)$ & $0.62(0.48,0.79)$ \\
\hline ANX: High & $0.71(0.65,0.77)$ & $0.65(0.57,0.73)$ & $0.76(0.70,0.83)$ & $0.74(0.51,1.06)$ \\
\hline ANX: Very high & $0.69(0.63,0.75)$ & $0.64(0.53,0.76)$ & $0.73(0.66,0.80)$ & $0.62(0.38,1.0)$ \\
\hline $\begin{array}{l}\text { Self injurious ideation } \\
\text { or attempt }\end{array}$ & $1.46(1.34,1.60)$ & $1.25(1.16,1.36)$ & $1.75(1.56,1.97)$ & $1.55(1.17,2.06)$ \\
\hline Behaviour symptom & $1.36(1.27,1.44)$ & $1.39(1.27,1.52)$ & $1.31(1.23,1.39)$ & \\
\hline $\begin{array}{l}\text { Problematic sexual } \\
\text { behaviour }\end{array}$ & $1.39(1.32,1.46)$ & $1.13(1.02,1.24)$ & $1.51(1.44,1.58)$ & \\
\hline Cognitive problem & $0.70(0.64,0.77)$ & $0.65(0.58,0.72)$ & $0.77(0.70,0.85)$ & $0.66(0.52,0.84)$ \\
\hline Victim of abuse & $1.27(1.20,1.34)$ & $1.16(1.09,1.23)$ & $1.34(1.26,1.43)$ & $1.46(1.19,1.78)$ \\
\hline $\begin{array}{l}\text { No Strong family } \\
\text { relationship }\end{array}$ & $1.24(1.17,1.30)$ & $1.31(1.22,1.41)$ & $1.20(1.13,1.27)$ & \\
\hline $\begin{array}{l}\text { Parental } \\
\text { addiction/substance } \\
\text { abuse }\end{array}$ & $1.45(1.38,1.52)$ & $1.48(1.37,1.61)$ & $1.40(1.32,1.47)$ & $1.35(1.11,1.65)$ \\
\hline Parent with $\mathrm{MH}$ issue & & & $1.07(1.02,1.23)$ & \\
\hline
\end{tabular}

\section{Discussion}

This study demonstrates the clinical complexity of youth who use substances and those who may require substance use treatment in the community and residential clinical settings across Ontario, Canada. Interestingly, the pattern of substance use among youth 
in community mental health setting is different than those in the general population. For instance, among youth in grades 7 to 12 in a general Canadian population sample, 27\% reported current use (use in the last 30 days) of alcohol, 19\% reported current cannabis use, and $8 \%$ reported other drugs [9], while this study shows that $11 \%$ of youth in the community mental health settings reported current alcohol use. Furthermore, the proportion of youth who reported use of illicit drugs is lower in the general population ( $8 \%$ ) than community mental health (interRAI ChYMH) sample (17\%), and $22 \%$ of youth in the community sample reported current use of any substance (including alcohol, cannabis, illicit drugs, and misuse of prescribed or over the counter medications). The nature of the items on the interRAI ChYMH-Screener, representing the majority of the sample, limited the ability to assess lifetime use of substances and alcohol. As might be expected, results from the residential sample confirmed that substance use is much more common among youth experiencing severe mental health concerns than those in the community sample in this study and general population samples from prior literature.

Understanding the reasons for substance use among youth can be challenging, as suggested by the biopsychosocial model of substance use and the wide range of psychosocial factors associated with substance use [14,16]. Although the results of this study are cross-sectional, the findings do offer insights into a range of circumstances that may be related to youth substance use. This study found that individual, familial, social, and environmental factors contribute to the risk of substance use among youth. Most factors associated with substance use were similar across clinical settings. This study found that several clinical variables that were statistically significant in the community were not significant for the residential sample. These included time since contact with CMHA, Hyperactivity/Distractibility symptoms, sleep problems, problematic sexual behavior, and having a parent with a mental health problem. However, we could not examine these differences extensively due to a lack of statistical power of the small sample size in the residential sample. Also, some of the differences may be due to the rarity of the clinical symptoms among children and youth (e.g., problematic sexual behavior). Furthermore, the difference may be related to the case-mix of youth admitted into residential care. For instance, barriers regarding access to care in the community may delay timely help-seeking resulting in complicated cases in need of urgent care or direct admission to residential care.

This study found that females (60.17\% of the sample population) were more commonly assessed compared to males (39.83\%) in the community settings, but more males (53.9\%) than females $(46.1 \%)$ in the residential settings. While there was no significant difference in the proportion of males and females who used any substance in community and residential settings, this study showed that females were more likely than males to report alcohol use and misuse of prescription or over-the-counter medication. In contrast, males were more likely than females to use illicit drugs. In the community sample for this study, the proportion of youth using substances was almost identical between males and females. However, after controlling for other variables, the relative risk of substance use was significantly lower among females compared to males. While the patterns of substance use were similar by sex, it is crucial to consider other unique factors related to substance use among youth of each sex. For example, we know that the brain, hormones, and metabolic systems differ between males and females, such as differences in body weight and metabolism of substances such as alcohol [64]. These differences may relate to the experiences and effects induced by drugs among each sex. For instance, the differences in the subjective effects (adverse or rewarding) experienced by males and females may affect the continued use of substances. Also, males are more likely than females to engage in risky behaviors, including substance use, in anticipation of perceived social rewards or peers' influence. Other factors that could be influencing this association include parental factors like gender, caregiving capacity, and substance use or addiction. This finding is important to consider within clinical practice and public health. For instance, substance use screening and counseling could be mandatory for prevention and early interventions for boys. 
This study shows that substance use was common, particularly among those of older age and in residential settings. While the pattern of substance use by age was similar for male and female youths in the community and residential settings, the proportion of substance use by age categories increased more for females than males in the residential settings. The relationship between age and substance use observed in this study was not surprising but underscored the potential utility of this study's findings for early intervention. Previous studies have also shown a strong association between the onset of substance use and the development of substance use disorder later in life [65]. For instance, excessive and early initiation of substances like cannabis increases the risk of schizophrenia among young adults [65]. This association highlights the importance of early prevention efforts to reduce potential risky behaviors and long-term adverse health effects. The finding that a subset of youth aged 12-14 used substances shows that prevention and intervention should also target younger age groups (i.e., less than 12 years).

The finding that parental mental health status was related to substance use reinforces prior literature demonstrating strong associations between genetic/familial factors and substance use $[66,67]$. Therefore, early and comprehensive interventions among youth with a history of parental substance issues could prevent future use. For instance, children of alcohol-dependent parents were more likely to use a substance [68,69]. While children of parents deficient in alcohol dehydrogenase enzyme and dopamine D2A1 gene are less likely to use the substance. Environmental factors like parental substance use and permission of substance use in the home increase the likelihood of offspring substance use. Parents that use substances could be modeling this behavior to their offspring or permit them to use substances under their supervision. Parents with substance use disorder may also lack the capacity to supervise or adequately parent their children. Therefore, care planning for this population should be based on multidimensional approaches that focus on the importance of intervening across multiple levels of socialization, including parents. For instance, parents should be engaged in the care planning and treatment of youth at risk or with substance use issues.

The results of this study reinforce prior theories on the roles that psychosocial factors like family and school experience play in substance use among youth, emphasizing the importance of their engagement in the prevention of substance use among young people. Strong parental/family support was found to be a protective factor against substance use among youth in this study, as were positive school experience and academic achievement. This study shows that even youth who were not at risk of disrupted education because they were homeschooled were more likely to use substances than those in school and at risk of disrupted education. This finding shows the importance that school enrollment may play in substance use. Perhaps the school environment provides social structure and engagement and a network for monitoring youth health behaviors even in light of existing mental health concerns. There is a need for further research on this finding.

Strong clinical predictors of substance use among this study sample include Hyperactive/Distraction, having a history of self-injurious ideation/attempt, and exposure to or experienced traumatic life events (physical, sexual, and emotional abuse). Substance use may precede psychopathologies, as youth who use substances may engage in risky behavior that increases their risk of experiencing trauma leading to PTSD [70]. Psychopathology may precede youth substance use. For instance, youth with a behavioral or psychological problem may have impaired learning or impulsivity, leading to an inability to conceptualize the consequences of substance use. They may also be experiencing school failure leading to associations with deviant peers and self-medication. Also, the association between youth substance use and these clinical predictors may be due to shared vulnerability of genetic predisposition and childhood psychosocial factors. However, in contrast to some previous studies, anxiety symptoms and cognitive impairment were associated with reduced risk of substance use among male and female youths in the community sample after controlling for other factors. 


\section{Limitations}

This study is cross-sectional; therefore, these results should be interpreted with care since it did not test temporality or establish causality. Also, study sample selection was biased since only those referred to service providers were assessed. Furthermore, this study examined a small sample size for the residential sample, limiting the confidence of this study's conclusions about the findings. In addition, this study did not examine peer support which is another important factor considered in youth substance use. There is also no data on tobacco use or the types, dose, mode of administration, and frequency of substance use. In addition, this study did not examine the association between gender (socially determined roles) and substance use or the association between the sex of youth with substance use conditions and the treatment they receive. Finally, this study did not examine family structure or the sex of the parent/caregiver who used substances to see if the pattern is similar (i.e., the male to female ratio of parents/caregivers who use substances).

\section{Implications for Research and Practice}

There are some important opportunities for future research to build upon from this study's findings. Given the cross-sectional nature of this study, there is a need to develop prospective cohort studies to examine how early childhood factors and experiences may relate to the initiation or continuation of substance use, particularly following first contact with health services. The broad use of the interRAI ChYMH in several jurisdictions will afford opportunities to identify these cohorts and examine changes and outcomes over time in relation to substance use. Further information about the dose and frequency of substance use will enhance the rigor of these longitudinal studies, such as using the interRAI Addictions Supplement to the ChYMH instrument. Qualitative studies will complement these longitudinal studies to explore with youth, and their families or caregivers, reasons for substance use and factors that they feel could be leveraged to prevent future substance use.

There are several opportunities for future research to address limitations in the sex and gender analysis of substance use in this study. Considering this study's patient type and setting (mental health), patterns of substance use by sex may have been subject to other factors not measured in this study (e.g., types of drugs). For instance, this study did not examine the association between gender (socially determined roles) and substance use or the association between the sex of youth with substance use conditions and the treatment they receive. Finally, this study did not examine family structure or the sex of the parent/caregiver who uses substances to see if the pattern is similar (i.e., the male to female ratio of parents/caregivers who use substances).

The different pattern of substance use among youth in the community and residential settings shows that care providers could intensify their efforts to identify and treat substance use conditions (initiation or disorder) among youth with less severe symptoms at first contact in all settings. Furthermore, based on the differences identified in the residential and community settings, treatments in the community setting could focus more on early identification, intervention, and monitoring, while the focus of treatment in the residential setting may be more about in-patient treatment and harm reduction. Also, comprehensive mental health assessment, including substance use assessment, should be completed for all youths seen in outpatient or residential settings at first contact. Substance use should also be considered within mental health treatment. Failure to treat mental health conditions that co-occur with substance use conditions and vice versa due to fragmented services would increase the burden on youth seeking treatment, leading to intensive treatment needs and premature termination, resulting in poor outcomes. 


\section{Conclusions}

Individual and parental factors increase youth's risk of substance use, highlighting the importance of a holistic approach to prevention/risk reduction, risk assessment, management, and recovery. There is no single cause of problematic substance use among youth. This study shows that exposure to traumatic life events, history of self-injurious ideation, parental substance use, and negative school experiences are strong predictors of substance use among youth. In contrast, a strong and supportive relationship with family is a protective factor. These results show that psychopathology and social factors (especially family and school) are extremely important in preventing and treating substance use among youth. Therefore, the prevention of substance use will require collaboration among many sectors of society. A comprehensive approach may focus on access to social and mental health supports, supportive education, reduced availability, and marketing restrictions.

Author Contributions: Conceptualization, O.O.A.; methodology, O.O.A. and S.L.S.; formal analysis, O.O.A.; investigation, O.O.A.; writing-original draft preparation, O.O.A.; writing-review and editing, C.P., S.L.S. and J.P.H.; visualization, O.O.A.; supervision, C.P.; project administration, O.O.A. and S.L.S.; funding acquisition, C.P. and J.P.H. All authors have read and agreed to the published version of the manuscript.

Funding: This research was funded by the Kitchener Waterloo Community Foundation Community Fund, Alice (Braley) Judges-Walter Judges Fund (https: / / www.kwcf.ca/fund-listing, accessed on 26 December 2021).

Institutional Review Board Statement: The study was conducted according to the guidelines of the Tri-Council Policy Statement for the Ethical Conduct for Research Involving Humans (TCPS, 2nd edition), Internalization Conference on Harmonization: Good Clinical Practice (ICH-GCP), the Ontario Personal Health Information Protection Act (PHIPA), and the applicable laws and regulations of the province of Ontario, and approved by the University of Waterloo Research Ethics Committees (protocol code 42649, 24 November 2020).

Informed Consent Statement: Consent was not obtained because this study involved analysis of secondary non-identified data.

Data Availability Statement: Restrictions apply to the availability of these data. Like other ethics proposals using interRAI data (e.g., ORE 30173), data will be retained indefinitely in a secure location that is the interRAI Canada data server at the University of Waterloo. As part of the licensing agreement within interRAI, all instruments are provided to agencies, organizations and facilities free of charge. In return for the use of the instruments, data from each agency, organization de-identified data is given to interRAI to improve instruments, develop quality indicators, case mix system sand evidence-informed care planning initiatives to improve the care of vulnerable populations. However, for this study, investigators would only have access to the data for the duration of this project.

Conflicts of Interest: The authors declare no conflict of interest. The funders had no role in the design of the study; in the collection, analyses, or interpretation of data; in the writing of the manuscript, or in the decision to publish the results. 


\section{Appendix A}

Table A1. Characteristics of ChYMH and ChYMH-S Samples.

\begin{tabular}{|c|c|c|c|c|c|}
\hline \multirow[b]{2}{*}{ Characteristics } & \multirow[b]{2}{*}{ Level } & \multicolumn{2}{|c|}{ ChYMH-Screener $(\mathrm{N}=38976)$} & \multicolumn{2}{|c|}{ ChYMH (N = 9142) } \\
\hline & & Freq & $\%$ & Freq & $\%$ \\
\hline \multirow[t]{2}{*}{ Patient type } & Community & 38,972 & 99.99 & 8446 & 92.4 \\
\hline & Residential & 4 & 0.01 & 696 & 7.6 \\
\hline \multirow[t]{2}{*}{ Sex } & Male & 15,099 & 38.4 & 4068 & 44.5 \\
\hline & Female & 23,877 & 61.3 & 5074 & 55.5 \\
\hline \multirow[t]{3}{*}{ Age group } & $12-14$ & 11,022 & 28.3 & 2779 & 30.4 \\
\hline & $15-16$ & 14,941 & 38.3 & 3510 & 38.4 \\
\hline & $17-18$ & 13,013 & 33.4 & 2853 & 31.2 \\
\hline \multirow[t]{3}{*}{$\begin{array}{l}\text { Risk of disrupted } \\
\text { education }\end{array}$} & Yes & 23,280 & 60 & 4717 & 52.7 \\
\hline & No & 15,145 & 39 & 4027 & 45 \\
\hline & Not applicable & 392 & 1 & 212 & 2.4 \\
\hline \multirow[t]{2}{*}{$\begin{array}{l}\text { No Strong and } \\
\text { supportive relationship } \\
\text { with family }\end{array}$} & Yes & 6727 & 17.3 & 2053 & 22.5 \\
\hline & No & 32,249 & 82.7 & 7089 & 77.5 \\
\hline \multirow[t]{2}{*}{$\begin{array}{l}\text { Parent/caregiver has } \\
\text { mental health issue }\end{array}$} & Yes & 13,536 & 34.7 & 3963 & 43.3 \\
\hline & No & 25,440 & 65.3 & 5179 & 56.7 \\
\hline \multirow[t]{2}{*}{$\begin{array}{l}\text { Parental } \\
\text { addiction/substance } \\
\text { use }\end{array}$} & Yes & 7691 & 19.7 & 2125 & 23.2 \\
\hline & No & 31,285 & 80.3 & 7017 & 76.8 \\
\hline \multirow[t]{4}{*}{$\begin{array}{l}\text { Highest number of } \\
\text { alcohol drinks in the } \\
\text { last (14-30 days) }\end{array}$} & None & 34,723 & 89.1 & 8059 & 88.2 \\
\hline & 1 & 1092 & 2.8 & 277 & 3 \\
\hline & $2-4$ & 1729 & 4.4 & 476 & 5.2 \\
\hline & $\geq 5$ & 1432 & 3.7 & 330 & 3.6 \\
\hline \multirow[t]{2}{*}{$\begin{array}{l}\text { Intentional misuse of } \\
\text { prescription or OTC } \\
\text { medication }\end{array}$} & Yes & 1197 & 3.1 & 502 & 5.5 \\
\hline & No & 37,779 & 96.9 & 8640 & 94.5 \\
\hline \multirow[t]{2}{*}{$\begin{array}{l}\text { Use of illicit drugs (in } \\
\text { the last } 14-30 \text { days) }\end{array}$} & Yes & 6568 & 16.9 & 1711 & 18.7 \\
\hline & No & 32,408 & 83.1 & 7431 & 81.3 \\
\hline \multirow[t]{2}{*}{$\begin{array}{l}\text { Use of any substance in } \\
\text { the last } 14-30 \text { days }\end{array}$} & Yes & 8583 & 22 & 2263 & 24.8 \\
\hline & No & 30,393 & 78 & 6879 & 75.2 \\
\hline \multirow[t]{2}{*}{ Behaviour symptom } & Yes & 20,654 & 53 & 5939 & 65 \\
\hline & No & 18,322 & 47 & 3203 & 35 \\
\hline \multirow[t]{2}{*}{$\begin{array}{l}\text { Self-injurious ideation } \\
\text { or attempt }\end{array}$} & Yes & 22,548 & 57.9 & 5515 & 60.3 \\
\hline & No & 16,428 & 42.1 & 367 & 39.8 \\
\hline \multirow[t]{2}{*}{$\begin{array}{l}\text { Problematic sexual } \\
\text { behaviour }\end{array}$} & Yes & 2332 & 6 & 425 & 4.7 \\
\hline & No & 36,644 & 94 & 8717 & 95.3 \\
\hline \multirow[t]{2}{*}{ Victim of abuse } & Yes & 13,875 & 35.6 & 3785 & 41.4 \\
\hline & No & 25,101 & 64.4 & 5357 & 58.6 \\
\hline
\end{tabular}


Table A1. Cont.

\begin{tabular}{|c|c|c|c|c|c|}
\hline \multirow[b]{2}{*}{$\begin{array}{l}\text { Distraction and } \\
\text { hyperactivity scale }\end{array}$} & \multirow[b]{2}{*}{ 0-None } & \multicolumn{2}{|c|}{ ChYMH-Screener (N = 38976) } & \multicolumn{2}{|c|}{ ChYMH (N = 9142) } \\
\hline & & 8061 & 20.7 & 1571 & 17.2 \\
\hline & 1-Low & 23,262 & 59.7 & 5204 & 56.9 \\
\hline & 2-Moderate & 3010 & 7.7 & 770 & 8.4 \\
\hline & 3-High & 2329 & 6 & 772 & 8.4 \\
\hline & 4-Very high & 2314 & 6 & 825 & 9 \\
\hline \multirow[t]{5}{*}{ Anxiety scale } & 0-None & 6729 & 17.3 & 1576 & 17.2 \\
\hline & 1-Low & 11,138 & 28.6 & 2315 & 25.3 \\
\hline & 2-Moderate & 13,498 & 34.6 & 2981 & 32.6 \\
\hline & 3-High & 6178 & 15.9 & 1766 & 19.3 \\
\hline & 4-Very high & 1433 & 3.7 & 504 & 5.5 \\
\hline \multirow[t]{5}{*}{$\begin{array}{l}\text { Depression severity } \\
\text { Index }\end{array}$} & 0-None & 1467 & 3.8 & 494 & 5.4 \\
\hline & 1-Low & 16,739 & 43 & 3751 & 41 \\
\hline & 2-Moderate & 10,490 & 26.9 & 2361 & 25.8 \\
\hline & 3-High & 4374 & 11.2 & 1049 & 11.5 \\
\hline & 4-Very high & 5906 & 15.1 & 1487 & 16.3 \\
\hline \multirow[t]{2}{*}{ Sleep problem } & Yes & 24,797 & 63.6 & 5486 & 60 \\
\hline & No & 14,179 & 36.4 & 3656 & 40 \\
\hline \multirow[t]{2}{*}{ Cognitive problem } & Yes & 5908 & 15.2 & 2672 & 29.2 \\
\hline & No & 33,068 & 84.8 & 6470 & 70.8 \\
\hline \multirow[t]{3}{*}{$\begin{array}{l}\text { Last contact with } \mathrm{CMH} \\
\text { agency in the last year }\end{array}$} & No contact & 13,301 & 34.1 & 1763 & 19.3 \\
\hline & $\geq 31$ & 8634 & 22.1 & 2416 & 26.5 \\
\hline & $\leq 30$ & 17,041 & 43.7 & 4953 & 54.2 \\
\hline
\end{tabular}

\section{References}

1. Pearson, C.; Janz, T.; Ali, J. Health at a Glance: Mental and Substance Use Disorders in Canada; Statistics Canada: Ottawa, ON, Canada, 2013.

2. Gray, K.M.; Squeglia, L.M. Research Review: What have we learned about adolescent substance use? J. Child Psychol. Psychiatry 2018, 59, 618-627. [CrossRef] [PubMed]

3. Sussman, S.; Skara, S.; Ames, S.L. Substance abuse among adolescents. Subst. Use Misuse 2008, 43, 1802-1828. [CrossRef] [PubMed]

4. Weinberg, N.Z.; Rahdert, E.; Colliver, J.D.; Glantz, M.D. Adolescent substance abuse: A review of the past 10 years. J. Am. Acad. Child Adolesc. Psychiatry 1998, 37, 252-261. [CrossRef]

5. United Nations Office on Drugs and Crime (UNODC). World Drug Report 2020 Drug Use and Consequences; United Nations Office on Drugs and Crime: Vienna, Austria, 2020.

6. Canadian Institutes of Health Research. COMPASS 2018/2019; Canadian Institutes of Health Research: Ottawa, ON, Canada, 2019.

7. Public Health Agency of Canada Preventing Problematic Substance Use in Youth: The Chief Public Health Officer's Report on The State of Public Health in Canada 2018. Available online: https://www.canada.ca/content/dam/phacaspc/documents / corporate/publications / chief-public-health-officer-reports-state-public-health-canada /2018-preventingproblematic-substance-use-youth/2018-preventing-problematic-substance-use-youth.pdf (accessed on 5 July 2020).

8. Canadian Centre on Substance Use and Addiction. Substance Abuse in Canada: Youth in Focus. Available online: https: / / www.ccsa.ca/substance-abuse-canada-youth-focus (accessed on 16 August 2020).

9. Leatherdale, S.T.; Burkhalter, R. The substance use profile of Canadian youth: Exploring the prevalence of alcohol, drug and tobacco use by gender and grade. Addict. Behav. 2012, 37, 318-322. [CrossRef] [PubMed]

10. Chadi, N.; Bagley, S.M.; Hadland, S.E. Addressing Adolescents' and Young Adults' Substance Use Disorders. Med. Clin. North Am. 2018, 102, 603-620. [CrossRef] [PubMed]

11. Esmaeelzadeh, S.; Moraros, J.; Thorpe, L.; Bird, Y. Examining the Association and Directionality between Mental Health Disorders and Substance Use among Adolescents and Young Adults in the U.S. and Canada-A Systematic Review and Meta-Analysis. J. Clin. Med. 2018, 7, 543. [CrossRef]

12. Bugbee, B.A.; Beck, K.H.; Fryer, C.S.; Arria, A.M. Substance Use, Academic Performance, and Academic Engagement among High School Seniors. J. Sch. Health 2019, 89, 145. [CrossRef]

13. Fergusson, D.M.; Boden, J.M. Cannabis use and later life outcomes. Addiction 2008, 103, 969-976. [CrossRef] 
14. Skewes, M.C.; Gonzalez, V.M. The Biopsychosocial Model of Addiction. In Principles of Addiction; Elsevier: Amsterdam, The Netherlands, 2013; pp. 61-70. [CrossRef]

15. Wall, T.L.; Ehlers, C.L. Genetic Influences Affecting Alcohol Use Among Asians. Alcohol Health Res. World 1995, $19,184-189$.

16. Trucco, E.M. A Review of Psychosocial Factors Linked to Adolescent Substance Use. Pharmacol. Biochem. Behav. 2020, 196, 172969. [CrossRef]

17. Herz, V.; Franzin, N.; Huemer, J.; Mairhofer, D.; Philipp, J.; Skala, K. Substance use and misuse among children and youth with mental illness A pilot study. Neuropsychiatrie 2018, 32, 18-25. [CrossRef] [PubMed]

18. Kaminer, Y.; Connor, D.F.; Curry, J.F. Comorbid Adolescent Substance Use and Major Depressive Disorders: A Review. Psychiatry 2007, 4, 32. [PubMed]

19. Bountress, K.; Chassin, L.; Lemery-Chalfant, K. Parent and peer influences on emerging adult substance use: A genetically informed study. Dev. Psychopathol. 2017, 29, 121-142. [CrossRef] [PubMed]

20. Henneberger, A.K.; Mushonga, D.R.; Preston, A.M. Peer Influence and Adolescent Substance Use: A Systematic Review of Dynamic Social Network Research. Adolesc. Res. Rev. 2020, 1, 3. [CrossRef]

21. Gudonis, L.C.; Lewis, L.; Tong, Y.; Tu, W.; Aalsma, M.C. Adolescent Romantic Couples Influence on Substance Use in Young Adulthood. J. Adolesc. 2012, 35, 638. [CrossRef]

22. Selnow, G.W.; Crano, W.D. Formal vs informal group affiliations: Implications for alcohol and drug use among adolescents. J. Stud. Alcohol 1986, 47, 48-52. [CrossRef]

23. Verkooijen, K.T.; De Vries, N.K.; Nielsen, G.A. Youth crowds and substance use: The impact of perceived group norm and multiple group identification. Psychol. Addict. Behav. 2007, 21, 55-61. [CrossRef]

24. Barnes, G.E.; Mitic, W.; Leadbeater, B.; Dhami, M.K. Risk and Protective Factors for Adolescent Substance Use and Mental Health Symptoms. Can. J. Community Ment. Health 2009, 28, 1-15. [CrossRef]

25. Belcher, H.M.E.; Shinitzky, H.E. Substance abuse in children: Prediction, protection, and prevention. Arch. Pediatr. Adolesc. Med. 1998, 152, 952-960. [CrossRef]

26. Stone, A.L.; Becker, L.G.; Huber, A.M.; Catalano, R.F. Review of risk and protective factors of substance use and problem use in emerging adulthood. Addict. Behav. 2012, 37, 747-775. [CrossRef]

27. Stephens, J.R.; Heffner, J.L.; Adler, C.M.; Blom, T.J.; Anthenelli, R.M.; Fleck, D.E.; Welge, J.A.; Strakowski, S.M.; Delbello, M.P. Risk and protective factors associated with substance use disorders in adolescents with first-episode mania. J. Am. Acad. Child Adolesc. Psychiatry 2014, 53, 771-779. [CrossRef] [PubMed]

28. Patton, G.C.; Sawyer, S.M.; Santelli, J.S.; Ross, D.A.; Afifi, R.; Allen, N.B.; Arora, M.; Azzopardi, P.; Baldwin, W.; Bonell, C.; et al. Our future: A Lancet commission on adolescent health and wellbeing. Lancet 2016, 387, 2423-2478. [CrossRef]

29. Patton, G.C.; Coffey, C.; Romaniuk, H.; Mackinnon, A.; Carlin, J.B.; Degenhardt, L.; Olsson, C.A.; Moran, P. The prognosis of common mental disorders in adolescents: A 14-year prospective cohort study. Lancet 2014, 383, 1404-1411. [CrossRef]

30. Collishaw, S. Annual research review: Secular trends in child and adolescent mental health. J. Child Psychol. Psychiatry 2015, 56, 370-393. [CrossRef]

31. Kessler, R.C.; Amminger, G.P.; Aguilar-Gaxiola, S.; Alonso, J.; Lee, S.; Üstün, T.B. Age of onset of mental disorders: A review of recent literature. Curr. Opin. Psychiatry 2007, 20, 359-364. [CrossRef]

32. Sharma, A.; Morrow, J.D. Neurobiology of Adolescent Substance Use Disorders. Child Adolesc. Psychiatr. Clin. North Am. 2016, 25, 367-375. [CrossRef]

33. Buchman, D.Z.; Skinner, W.; Illes, J. Negotiating the relationship between addiction, ethics, and brain science. AJOB Neurosci. 2010, 1, 36-45. [CrossRef]

34. Tompsett, C.J.; Domoff, S.E.; Toro, P.A. Peer Substance Use and Homelessness Predicting Substance Abuse from Adolescence Through Early Adulthood. Am. J. Community Psychol. 2013, 51, 520-529. [CrossRef]

35. Van Ryzin, M.J.; Fosco, G.M.; Dishion, T.J. Family and peer predictors of substance use from early adolescence to early adulthood: An 11-year prospective analysis. Addict. Behav. 2012, 37, 1314-1324. [CrossRef]

36. Eme, R. Male Adolescent Substance Use Disorder and Attention-Deficit Hyperactivity Disorder: A Review of the Literature. ISRN Addict. 2013, 2013, 1-10. [CrossRef]

37. Wilson, J.J.; Levin, F.R. Attention Deficit Hyperactivity Disorder (ADHD) and Substance Use Disorders. Curr. Psychiatry Rep. 2001, 3, 497-506. [CrossRef] [PubMed]

38. Poorolajal, J.; Haghtalab, T.; Farhadi, M.; Darvishi, N. Substance use disorder and risk of suicidal ideation, suicide attempt and suicide death: A meta-analysis. J. Public Health 2016, 38, e282-e291. [CrossRef] [PubMed]

39. Rush, B.; Urbanoski, K.; Bassani, D.; Castel, S.; Wild, T.C.; Strike, C.; Kimberley, D.; Somers, J. Prevalence of Co-Occurring Substance Use and other Mental Disorders in the Canadian Population. Can. J. Psychiatry 2008, 53, 800-809. [CrossRef] [PubMed]

40. Wilens, T.E.; Biederman, J.; Abrantes, A.M.; Spencer, T.J. Clinical characteristics of psychiatrically referred adolescent outpatients with substance use disorder. J. Am. Acad. Child Adolesc. Psychiatry 1997, 36, 941-947. [CrossRef] [PubMed]

41. ICES. MHASEF Report 2015. Available online: https://www.ices.on.ca/flip-publication/MHASEF_Report_2015/files/assets/ basic-html/index.html\#1 (accessed on 21 December 2020).

42. Rachbeiscl, J.; Scott, J.; Dixon, L. Co-Occurring severe mental illness and substance use disorders: A review of recent research. Psychiatr. Serv. 1999, 50, 1427-1434. [CrossRef] [PubMed] 
43. Drake, R.E.; Mueser, K.T.; Clark, R.E.; Wallach, M.A. The course, treatment, and outcome of substance disorder in persons with severe mental illness. Am. J. Orthopsychiatry 1996, 66, 42-51. [CrossRef]

44. Watson, G.K.; Carter, C.; Finlay, B.; Mlakar, M.; Brown, P.; Palinsky, S.; Davis, S.; Chaim, G.; Clark, H.; Analyst, P.; et al. Pathways to Care for Youth with Concurrent Mental Health and Substance Use Disorders; Ontario Centre of Excellence for Child and Youth Mental Health: Ottawa, ON, Canada, 2014.

45. Canadian Mental Health Association Residential Treatment for Children and Youth. Available online: https://ontario.cmha.ca/ documents/residential-treatment-for-children-and-youth / (accessed on 27 March 2021).

46. Canadian Mental Health Association Addiction and Substance Use Treatment Options. Available online: https://ontario.cmha. ca/addiction-and-substance-misuse-treatment-options/ (accessed on 27 March 2021).

47. Watson, G.K.; Carter, C.; Manion, I. Pathways to Care for Youth with Concurrent Mental Health and Substance Use Disorders. Available online: https:/ / www.cymh.ca/Modules / ResourceHub/?id=566257b1-a51f-493c-adcc-bd486492e236 (accessed on 27 March 2021).

48. Health Quality Ontario and the Institute for Clinical Evaluative Sciences. Taking Stock: A Report on the Quality of Mental Health and Addictions Services in Ontario; Health Quality Ontario: Toronto, ON, Canada, 2015; ISBN 9781460670590.

49. Wang, P.S.; Berglund, P.A.; Olfson, M.; Kessler, R.C. Delays in Initial Treatment Contact after First Onset of a Mental Disorder. Health Serv. Res. 2004, 39, 393. [CrossRef]

50. Government of Ontario. Roadmap to Wellness: A Plan to Build Ontario's Mental Health and Addictions System, Ontario.ca 2020. Available online: https:/ / www.ontario.ca/page/roadmap-wellness-plan-build-ontarios-mental-health-and-addictions-system (accessed on 26 December 2021).

51. Stewart, S.L.; Hirdes, J.P.; Curtin-Telegdi, N.; Perlman, C.M.; McKnight, M.; MacLeod, K.; Ninan, A.; Currie, M.; Carson, S.; Morris, J.N.; et al. InterRAI Child and Youth Mental Health (ChYMH) Assessment Form and User's Manual: For Use with In-Patient and Community-Based Assessments; Version 9.3; interRAI: Wellington, New Zealand, 2015.

52. Stewart, S.L.; Hamza, C.A. The Child and Youth Mental Health Assessment (ChYMH): An examination of the psychometric properties of an integrated assessment developed for clinically referred children and youth. BMC Health Serv. Res. 2017, 17, 82. [CrossRef]

53. Stewart, S.L.; Babcock, S.E. InterRAI Child and Youth Mental Health-Screener (ChYMH-S): A Psychometric Evaluation and Validation Study. Child Psychiatry Hum. Dev. 2020, 51, 769-780. [CrossRef]

54. Li, Y.; Babcock, S.E.; Stewart, S.L.; Hirdes, J.P.; Schwean, V.L. Psychometric Evaluation of the Depressive Severity Index (DSI) Among Children and Youth Using the interRAI Child and Youth Mental Health (ChYMH) Assessment Tool. Child Youth Care Forum 2021. [CrossRef]

55. Ballinger, G.A. Using Generalized Estimating Equations for Longitudinal Data Analysis. Organ. Res. Methods 2004, 7, 127-150. [CrossRef]

56. Hu, F.B.; Goldberg, J.; Hedeker, D.; Flay, B.R.; Pentz, M.A. Comparison of Population-Averaged and Subject-Specific Approaches for Analyzing Repeated Binary Outcomes. Am. J. Epidemiol. 1998, 147, 694-703. [CrossRef] [PubMed]

57. Agresti, A. Introduction to Categorical Data, 2nd ed.; John Wiley \& Sons: Hoboken, NJ, USA, 1996; ISBN 978-0-471-22618-5.

58. Leigh, J.P. Assessing the importance of an independent variable in multiple regression: Is stepwise unwise? J. Clin. Epidemiol. 1988, 41, 669-677. [CrossRef]

59. Hosmer, D.W.; Lemeshow, S.; Sturdivant, R.X.; Army Academy US. Applied Logistic Regression Third Edition. 2013. Available online: https:/ / www.wiley.com (accessed on 16 July 2021).

60. Evans, S.R.; Hosmer, D.W., Jr. Goodness of Fit Tests for Logistic GEE Models: Simulation Results. Commun. Stat. -Simul. Comput. 2004, 33, 247-258. [CrossRef]

61. Cui, J. QIC program and model selection in GEE analyses. Stata J. 2007, 7, 209-220. [CrossRef]

62. Hanley, J.A.; McNeil, B.J. The Meaning and Use of the Area under a Receiver Operating Characteristic (ROC) Curve1. Radiology 1982, 143, 29-36. [CrossRef]

63. Cook, N.R. Use and misuse of the receiver operating characteristic curve in risk prediction. Circulation 2007, 115, 928-935. [CrossRef]

64. McHugh, R.K.; Votaw, V.R.; Sugarman, D.E.; Greenfield, S.F. Sex and gender differences in substance use disorders. Clin. Psychol. Rev. 2018, 66, 12-23. [CrossRef]

65. Hanna, R.C.; Perez, J.M.; Ghose, S. Cannabis and development of dual diagnoses: A literature review. Am. J. Drug Alcohol Abus. 2017, 43, 442-455. [CrossRef]

66. Ducci, F.; Goldman, D. The Genetic Basis of Addictive Disorders. Psychiatr. Clin. North Am. 2012, 35, 495-519. [CrossRef]

67. Prom-Wormley, E.C.; Ebejer, J.; Dick, D.M.; Bowers, M.S. The genetic epidemiology of substance use disorder: A review. Drug Alcohol Depend. 2017, 180, 241-259. [CrossRef] [PubMed]

68. Glantz, M.D.; Chambers, J.C. Prenatal drug exposure effects on subsequent vulnerability to drug abuse. Dev. Psychopathol. 2006, 18, 893-922. [CrossRef] [PubMed]

69. Agrawal, A.; Scherrer, J.F.; Grant, J.D.; Sartor, C.E.; Pergadia, M.L.; Duncan, A.E.; Madden, P.A.F.; Haber, J.R.; Jacob, T.; Bucholz, K.K.; et al. The effects of maternal smoking during pregnancy on offspring outcomes. Prev. Med. 2010, 50, 13-18. [CrossRef] [PubMed]

70. Van den Brink, W. Substance use disorders, trauma, and PTSD. Eur. J. Psychotraumatology 2015, 6, 27632. [CrossRef] 